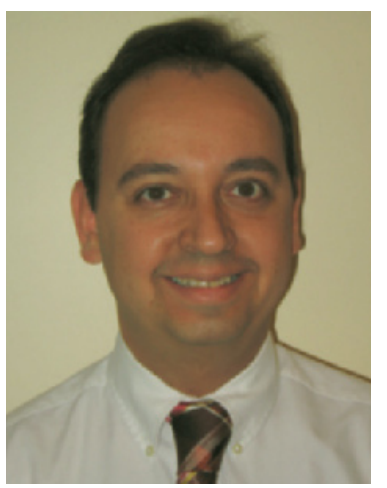

_J.L. Pinheiro-Franco Neurosurgeon Samaritano Hospital, São Paulo, Brazil

\section{Spondylolisthesis A clinico-radiological wide spectrum entity that must never be underestimated}

Spondylolisthesis, mainly the high-grade type, is among the most challenging deformities faced by spine surgeons. It combines sets of different clinical and radiological events, providing a formidable variety of stimulating cases. The present edition of ASN\&J concentrates on spondylolisthesis, reviews established concepts, analyses them and provides new insights learned from years of experience. A star studded cast of tenured authors offers, in the next pages, their view about 7 main topics.

The first one, by Drs. Winters and Herkowitz, provides a comprehensive overview on degenerative spondylolisthesis, navigating from epidemiology, pathophysiology, radiological and clinical evaluation until the management, therapeutic options, including the most recent evidence based information and insights on results with new technological devices. Dr. Herkowitz, a worldwide respected scientific authority in degenerative spondylolisthesis, included classical scientific articles as the ones from Jim Weinstein (editor of Spine - Phila PA 1976) published in N Engl J Med (2007) and in J Bone Joint Surg Am (2009, results of the SPORT trial).

The second chapter, by Drs. Bierry and Dietemann (Chairman of the Radiological Department of Strasbourg University, France) depicts a fully illustrated review of the most important imaging aspects of degenerative and isthmic spondylolisthesis. It is followed by the excellent and complementary contribution by Drs. Zukotynski et al. from Harvard Medical School in Boston. The authors discuss pertinent modern methods of imaging such as SPECT, pointing out the tools, advantages and disadvantages over each other.

Dr. Schlenzka et al., from Finland, in the text entitled "Low-grade isthmic spondylolisthesis in children, adolescents, and young adults", develop a comprehensive review of multiple aspects of the subject. The authors present the results of their long-standing research in Helsinki. Natural history, risk of progression, symptoms, imaging and therapeutic strategies, including their own criteria for treatment, results of extensive experience, are discussed with vast and high-quality evidence-based literature.

Drs. Mac-Thiong and Labelle from Montreal reveal that sacro-pelvic morphology and balance are strong determinants of sagittal spino-pelvic alignment but have not yet been included in any treatment algorithm. The authors expose their current understanding of sagittal spino-pelvic balance for the treatment of high-grade developmental spondylolisthesis, based on their experience and work over the past 10 years within the Spinal Deformity Study Group (SDSG). They offer an important revision of the present knowledge in sagittal balance, examine the SDSG classification for high-grade spondylolisthesis and demonstrate with illustrative cases its clinical relevance for the operative treatment. The sagittal balance analysis today is easily accessed by measuring the parameters or through spine softwares such as Optispine.

Finally, closing this edition, Drs. Stoker, Buchowski, Zebala, et al., who have decades of experience between them, provide a comprehensive review of their recent operative practice with high-grade spondylolisthesis. Indications and contraindications for surgery are explained and a complete analysis of the diverse surgical aspects is provided: exposure, decompression, instrumentation, release, reduction, fusion and alternatives are abundantly discussed. Our readers will be finally rewarded with five greatly illustrated cases.

I hope you will enjoy this overview as well as I did. Have a good reading! • 